\title{
Developmental Delay in Children with X-Linked Ichthyosis: A Case Series
}

\author{
Su Jeong Park, MD, Kyung Hee Park, MD, Mi Hye Bae, MD, Young Mi Kim, MD \\ Department of Pediatrics, Pusan National University Hospital, Pusan National University School of Medicine and Biomedical Research \\ Institute, Busan, Korea
}

Received: June 7, 2021

Revised: July 14, 2021

Accepted: July 21, 2021

Corresponding author:

Young Mi Kim, MD

Department of Pediatrics, Pusan

National University Hospital, 179

Gudeok-ro, Seo-gu, Busan 49241,

Korea

Tel: +82-51-240-7298

Fax: +82-51-248-6205

E-mail: pink2129@naver.com
X-linked ichthyosis (XLI; MIM \#308100) is a dermatologic disorder characterized by large, dark brown scales similar to fish scales. It starts primarily on the extensor surfaces and the side of the trunk symmetrically, becomes widely distributed, and then disappears throughout childhood. Most cases of XLI are caused by mutations in the steroid sulfatase (STS) gene (online Mendelian inheritance in men [OMIM] ${ }^{*}$ 300747), which is located on the short arm of the X chromosome at Xp22.3 and encodes the STS enzyme [1]. Most XLI patients (90\%) have large deletions within or including STS [2]. These patients display various clinical symptoms, as well as skin lesions. Baek and Aypar [3] reported a 5-year-old girl who presented with mild autism, attention-deficit hyperactivity disorder (ADHD), and dry, scaly skin on the body. A chromosomal microarray (CMA) revealed a large deletion, including STS and neuroligin 4, OMIM*300427 (NLGN4), associated with ADHD and autism [3]. Here, we report three children with XLI and developmental delay. We confirmed XLI and the presence of a large deletion through CMA with a single-nucleotide polymorphism (SNP) array and also investigated other associated clinical symptoms.

The clinical characteristics of the three patients and their CMA results are summarized in Table 1. Patient 1 was a 7 -year-old boy who was born at 35 weeks 6 days by cesarean section due to maternal preeclampsia. His birth weight was $1,886 \mathrm{~g}$ and he showed intrauterine growth retardation. He underwent surgery due to coarctation of aorta on day 6 after birth. At 12 months of age, he presented with a nevus on his forehead, dry and scaly skin, increased muscle tone, and global developmental delay. No family members had the typical skin lesions of XLI. Magnetic resonance imaging (MRI) of the brain and an electroencephalogram (EEG) were normal. The karyotype was normal. The patient received continued rehabilitation, but the developmental delay did not improve appreciably. He showed no sign of autism or ADHD. His full-scale intelligence quotient (FSIQ) was 35 to 40 and social quotient (SQ) was 37. CMA was performed with a CytoScan 750K array (Affymetrix, Santa Clara, CA, USA) according to the manufacturer's recommendations in all three patients. The SNP array revealed a deletion of approximately $1.7 \mathrm{Mb}$ at Xp22.31 (6,455,151-8,141,076). The deleted region included several genes, such as variably charged, X chromosome, 3A, OMIM*300533 (VCX3A), haloacid dehalogenase-like hydrolase domain-containing 1A, OMIM*306480 (HDH-

Copyright (C) 2021 Korean Child Neurology Society

This is an Open Access article distributed under the terms of the Creative Commons Attribution Non-Commercial License (http://creativecommons.org/licenses/by-nc/4.0/) which permits unrestricted non-commercial use, distribution, and reproduction in any medium, provided the original work is properly cited. 
Table 1. Clinical features and chromosomal microarray data of the three patients with X-linked ichthyosis and developmental delay

\begin{tabular}{|c|c|c|c|}
\hline Variable & Patient 1 & Patient 2 & Patient 3 \\
\hline Age at diagnosis & 7 years & 17 months & 3 years \\
\hline Sex & Male & Male & Female \\
\hline \multicolumn{4}{|l|}{ Phenotypes } \\
\hline \multicolumn{4}{|l|}{ Skin } \\
\hline Dry skin (HP:0000958) & + & + & + \\
\hline Ichthyosis (HP:0008064) & + & + & - \\
\hline \multicolumn{4}{|l|}{ Neurological } \\
\hline Cognitive impairment (HP:0100543) & + & + & + \\
\hline Delayed gross motor development (HP:0003294) & + & + & + \\
\hline Global developmental delay (HP:0001263) & + & + & + \\
\hline Epilepsy (HP:0001250) & + & - & - \\
\hline Infantile axial hypotonia (HP:0009062) & + & - & + \\
\hline \multicolumn{4}{|l|}{ Growth } \\
\hline Intrauterine growth retardation (HP:0001511) & + & + & - \\
\hline \multicolumn{4}{|l|}{ Eyes } \\
\hline Myopia (HP: 0000545) & + & - & - \\
\hline Strabismus (HP:0000486) & - & - & + \\
\hline \multicolumn{4}{|l|}{ Others } \\
\hline Coarctation of aorta (HP:0001680) & + & - & - \\
\hline Scoliosis (HP:0002650) & + & - & - \\
\hline Premature birth (HP:0001622) & + & - & - \\
\hline Brain magnetic resonance imaging & Normal & Normal & Normal \\
\hline Electroencephalography (first) & Normal & Normal & Normal \\
\hline Chromosome & $x$ & $x$ & $x$ \\
\hline Cytoregion & p22.31 & p22.31 & p22.31 \\
\hline Type & Loss & Loss & Loss \\
\hline Deletion size (bp) & 1685925 & 1653683 & 1669613 \\
\hline Deleted position & $6,455,151-8,141,076$ & $6,473,896-8,127,579$ & $6,458,939-8,143,509$ \\
\hline \multicolumn{4}{|l|}{ Deleted genes } \\
\hline VCX3A (0MIM * 300533) & + & + & + \\
\hline HDHD1A (OMIM * 306480) & + & + & + \\
\hline STS (OMIM * 300747) & + & + & + \\
\hline VCX $(0 \mathrm{MIM} *$ 300229) & + & + & + \\
\hline PNPLA4 (OMIM * 300102) & + & + & + \\
\hline
\end{tabular}

$H P$, human phenotype ontology identification number; VCX3A, variably charged, $X$ chromosome, 3A; OMIM, online Mendelian inheritance in men; HDHD1A, haloacid dehalogenase-like hydrolase domain-containing 1A; STS, steroid sulfatase; VCX, variably charged, $X$ chromosome; PNPLA4, patatin-like phospholipase domain-containing protein 4.

D1A), STS, VCX (OMIM * 300229), and patatin-like phospholipase domain-containing protein 4, OMIM*300102 (PNPLA4). The patient was diagnosed as having XLI with intellectual disability. At 9 years of age, he presented with general tonic seizures and focal interictal discharge over the occipital area on EEG at the onset of the seizure. We diagnosed the patient with epilepsy. The patient is now 11 years of age and has epilepsy, myopia, and scoliosis. He cannot speak any words and cannot sit or walk alone.

Patient 2 was a 21-month-old boy with dry and scaly skin since birth. He was born at term by cesarean section. His birth weight was $2,460 \mathrm{~g}$ and he showed intrauterine growth retardation. His maternal grandfather and a maternal cousin had ichthyosis. His parents noticed his global developmental delay at approximately 12 months of age. In the Korean Infant and Child Developmental Test, he showed global developmental delay, with a developmental age (DA) of gross motor skills of 12 months old (developmental quotient $[\mathrm{DQ}] 57$, normal $\mathrm{DQ}>85$ ), a DA of fine motor skills of 16 months old (DQ76), a DA of social-personal development of 12 months old (DQ 57), a DA of language development of 16 months old (DQ76), and a DA of cognitive function of 16 months 
old (DQ76). Brain MRI and an EEG scan were normal. Tests for inborn errors of metabolism were normal. He showed no signs of autism or ADHD. The SNP array revealed a deletion of approximately 1.7 Mb at Xp22.31 (6,473,896-8,127,579), which included VCX3A, HDHD1A, STS, VCX, and PNPLA4. He was diagnosed with XLI and global developmental delay. At the time of this report, the patient cannot walk alone and speak any significant words, but his height and weight are in the normal range.

Patient 3 was a 3-year-old girl born at term. She showed axial hypotonia, dry skin without scales, and global developmental delay during infancy. She underwent surgery for strabismus at 2 years of age. There was no family history of ichthyosis. Brain MRI and an EEG scan were normal. Tests for inborn errors of metabolism were normal. She showed no signs of autism or ADHD. Her FSIQ was 55 and SQ was 56. The SNP array revealed a deletion of approximately 1.7 Mb at Xp22.31 (6,458,939-8,143,509), which included VCX3A, HDHD1A, STS, VCX, and PNPLA4. She was diagnosed as being a carrier of XLI with a milder skin phenotype than that of male XLI, intellectual disability, and strabismus. She can now walk alone, but runs imperfectly. Her height and weight are in the normal range.

$\mathrm{XLI}$ is an X-linked recessive disorder that occurs almost exclusively in males. It has been rarely reported in homozygous women who were offspring of an affected man and a female carrier [4]. Approximately $90 \%$ of patients with XLI have a large deletion that includes STS. The remaining cases are caused by point mutations within STS. The encoded STS protein belongs to the sulfatase family. The enzyme hydrolyzes several 3-beta-hydroxysteroid sulfates, and specifically converts the sulfated form of dehydroepiandrosterone (DHEA), otherwise known as DHEAS, to DHEA. In rodents, increased blood levels of DHEAS and DHEA can result in enhanced memory [5]. In humans, lower levels of DHEA in the blood have been reported to be associated with diverse phenotypes, such as $\mathrm{ADHD}$, corneal opacities, cryptorchidism, epilepsy, and developmental delay [1]. Patients with STS escape from X-inactivation (lyonization) and heterozygous carriers have been reported to exhibit increased rates of neurodevelopmental disorders, elevated levels of inattention, impulsivity, autism-related traits, and general psychological distress [6]. Placental STS deficiency can occur during pregnancy in XLI fetuses of a carrier mother, and the risk of obstetric complications, such as delayed and prolonged labor, is higher than in the general population [1]. In the present report, patient 3 was a girl with a heterozygous deletion of Xp22.31 including STS. Although she displayed global developmental delay and intellectual disability, she showed a mild skin phenotype with only dry skin, not the typical scaly skin of XLI.

We present three cases of XLI with a deletion of approximately $1.7 \mathrm{Mb}$ at Xp22.31. All three patients presented with dry, scaly skin and developmental delay. The SNP array revealed large deletions that included STS, HDHD1A, VCX3A, VCX, and PNPLA4 (Fig. 1). HDHD1A encodes a member of the haloacid dehalogenase-like (HAD) hydrolase superfamily. The encoded protein has no known biological function. PNPLA4 encodes a member of the patatin-like family of phospholipases. The encoded enzyme has triacylglycerol lipase and transacylase activities, and it may be involved in adipocyte triglyceride homeostasis. It has been reported that PNPLA4 might be associated with autism [7]. Variable charge, $\mathrm{X}$-linked ( $V C X)$ belongs to the $V C X / Y$ family, which has multiple members on both the $\mathrm{X}$ and $\mathrm{Y}$ chromosome, and all are expressed exclusively in male germ cells. $V C X / Y$ genes encode small and highly charged proteins of unknown function. $V C X 3 A$, also known $V C X A$, has been reported to show an association with abnormal neurologic phenotypes. Fukami et al. [8] showed that VCXA was preserved in all XLI patients without developmental delay, whereas it was deleted in all XLI patients with developmental delay. Hosomi et al. [9] identified a VCX3A promoter deletion in a case of XLI with borderline mental retardation. The collective findings indicate that deletions of $V C X 3 A$ or STS might be associated with

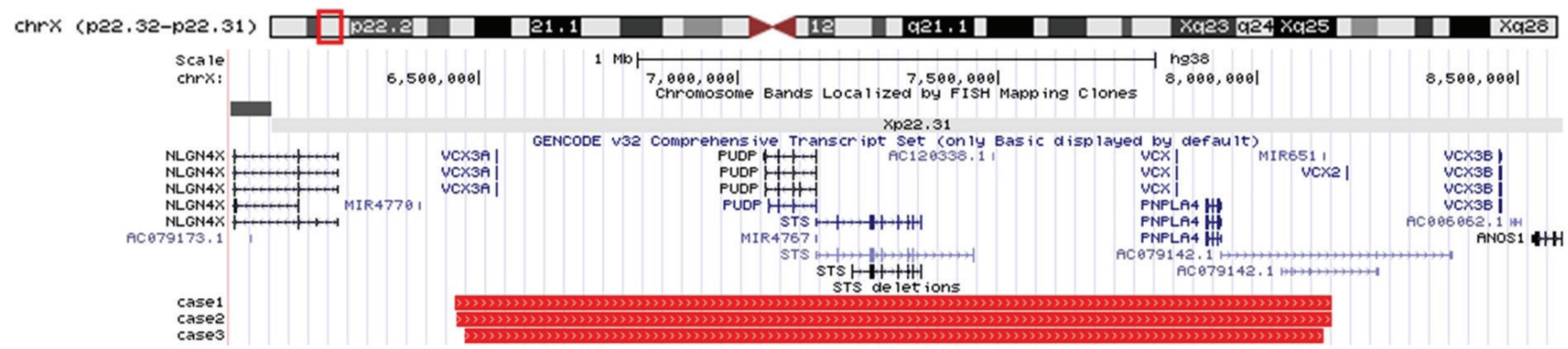

Fig. 1. X-linked ichthyosis cases identified by laboratory chromosomal microarray (CMA). University of California Santa Cruz genome browser image (genome build hg38) shows deletions in the three cases (red bars) identified by CMA, which include steroid sulfatase (STS; at Xp22.31; chrX:7,147,977-7,453,758). 
developmental delay in the XLI patients described in this report. We can assume that patients who have deletions of both $V C X 3 A$ and STS show a greater variety of phenotypes and more severe neurologic symptoms.

In conclusion, the three present cases, in combination with previous studies, indicate that XLI can be associated with developmental delay. Cytogenetic tests are an important tool to identify and predict the risk of neurologic manifestations. We believe that our report will improve awareness of the neurologic manifestations of XLI on the basis of cytogenetic tests.

This study was approved by the Institutional Review Board of Pusan National University Hospital (approval number: 2007-030093). Written informed consent was obtained from the parents of all patients.

\section{Conflicts of interest}

No potential conflict of interest relevant to this article was reported.

\section{ORCID}

Su Jeong Park, https://orcid.org/0000-0001-7496-7159

Young Mi Kim, https://orcid.org/0000-0002-6853-2228

\section{Author contribution}

Conceptualization: YMK. Data curation: SJP. Formal analysis: MHB. Funding acquisition: YMK. Methodology: KHP. Project administration: KHP. Visualization: MHB. Writing-original draft: SJP. Writing-review \& editing: YMK.

\section{References}

1. Fernandes NF, Janniger CK, Schwartz RA. X-linked ichthyosis: an oculocutaneous genodermatosis. J Am Acad Dermatol 2010;62:480-5.

2. Takeichi T, Akiyama M. Inherited ichthyosis: non-syndromic forms. J Dermatol 2016;43:242-51.

3. Baek WS, Aypar U. Neurological manifestations of X-linked ichthyosis: case report and review of the literature. Case Rep Genet 2017;2017:9086408.

4. Thauvin-Robinet C, Lambert D, Vaillant G, Caillier P, Donzel A, Cusin V, et al. X-linked recessive ichthyosis in a girl: strategy for identifying the causal mechanism. Br J Dermatol 2005;152: 191-3.

5. Reed MJ, Purohit A, Woo LW, Newman SP, Potter BV. Steroid sulfatase: molecular biology, regulation, and inhibition. Endocr Rev 2005;26:171-202.

6. Cavenagh A, Chatterjee S, Davies W. Behavioural and psychiatric phenotypes in female carriers of genetic mutations associated with X-linked ichthyosis. PLoS One 2019;14:e0212330.

7. Labonne JDJ, Driessen TM, Harris ME, Kong IK, Brakta S, Theisen J, et al. Comparative genomic mapping implicates LRRK2 for intellectual disability and autism at 12q12, and HDHD1, as well as PNPLA4, for X-linked intellectual disability at Xp22.31. J Clin Med 2020;9:274.

8. Fukami M, Kirsch S, Schiller S, Richter A, Benes V, Franco B, et al. A member of a gene family on Xp22.3, VCX-A, is deleted in patients with X-linked nonspecific mental retardation. Am J Hum Genet 2000;67:563-73.

9. Hosomi N, Oiso N, Fukai K, Hanada K, Fujita H, Ishii M. Deletion of distal promoter of VCXA in a patient with X-linked ichthyosis associated with borderline mental retardation. J Dermatol Sci 2007;45:31-6. 